PROCEEDINGS OF THE

AMERICAN MATHEMATICAL SOCIETY

Volume 136, Number 2, February 2008, Pages 539-548

S 0002-9939(07)08884-3

Article electronically published on November 3, 2007

\title{
STRONG CONVERGENCE RESULTS FOR NONSELF MULTIMAPS IN BANACH SPACES
}

\author{
N. SHAHZAD AND H. ZEGEYE \\ (Communicated by Joseph A. Ball)
}

\begin{abstract}
We prove strong convergence theorems for multimaps under mild conditions, which include Browder's convergence theorem as well as Reich's convergence theorem. We thus provide a partial answer to Jung's question.
\end{abstract}

\section{INTRODUCTION}

Let $D$ be a nonempty closed convex subset of a Banach space $E$ and let $C B(E)$, $K(E)$ and $K C(E)$ denote the family of nonempty closed bounded subsets, nonempty compact subsets and nonempty compact convex subsets of $E$, respectively. Let $H$ be the Hausdorff metric on $C B(E)$ and $T: D \longrightarrow C B(E)$ a multimap. Then $T$ is said to be a contraction if there exists $0 \leq k<1$ such that $H(T x, T y) \leq k\|x-y\|$ for $x, y \in D$. If $k=1$, then $T$ is called nonexpansive. A point $x^{*}$ is a fixed point of $T$, if $x^{*} \in T x^{*}$. The set of fixed points is denoted by $F(T)$.

Let $T: D \rightarrow K(D)$. Given a $u \in D$ and a $t \in(0,1)$, let $G_{t}: D \rightarrow K(D)$ be defined by

$$
G_{t} x=t T x+(1-t) u, \quad x \in D .
$$

Then $G_{t}$ is a contraction and admits a fixed point $x_{t} \in D$ by the Nadler contraction principle [16], that is,

$$
x_{t} \in t T x_{t}+(1-t) u \text {. }
$$

If $T$ is single-valued, then

$$
x_{t}=t T x_{t}+(1-t) u .
$$

In this case, $\left\{x_{t}\right\}$ is an approximating fixed point of $T$ since it has the property that if $\left\{x_{t}\right\}$ is bounded, then $\lim _{t \rightarrow 1}\left\|x_{t}-T x_{t}\right\|=0$.

The strong convergence of the net $\left\{x_{t}\right\}$ for a self or nonself nonexpansive singlevalued map $T$ has been investigated by Browder [3, Halpern [7, Jung and Kim 11, Kim and Takahashi 12, Reich 19, Singh and Watson 22, Takahashi and Kim [24, Xu 27, Xu and Yin 29, etc. For details on iterative methods, we refer to 2 .

In 1967, Browder [3] established the following strong convergence theorem.

Received by the editors June 21, 2006 and, in revised form, August 25, 2006.

2000 Mathematics Subject Classification. Primary 47H10, 47H09.

Key words and phrases. Nonexpansive multimap, fixed point, nonexpansive retract, Banach limit, inwardness, Banach space. 
Theorem B ([3]). Let $D$ be a nonempty closed bounded convex subset of a Hilbert space $E$ and $T$ a nonexpansive self-mapping of D. Let $\left\{t_{n}\right\}$ be a sequence in $(0,1)$ converging to 1 . Fix $u \in D$ and define a sequence $\left\{x_{n}\right\}$ in $D$ by

$$
x_{n}=t_{n} T x_{n}+\left(1-t_{n}\right) u, \quad n \in \mathbf{N} .
$$

Then $\left\{x_{n}\right\}$ converges strongly to the element of $F(T)$ nearest to $u$.

Reich [19] extended Browder's theorem.

Theorem $\mathbf{R}([19])$. Let $D$ be a nonempty closed bounded convex subset of a uniformly smooth Banach space $E$ and $T$ a nonexpansive self-mapping of $D$. Fix $u \in D$ and define a net $\left\{x_{t}\right\}$ in $D$ by

$$
x_{t}=t T x_{t}+(1-t) u, \quad t \in(0,1) .
$$

Then $\left\{x_{t}\right\}$ converges strongly as $t \rightarrow 1$ to the element of $F(T)$ nearest to $u$.

Pietramala [17] (see also Jung 9]) gave an example showing that Browder's theorem cannot be extended to the multivalued case without adding an extra assumption. López Acedo and $\mathrm{Xu}$ [15] established the strong convergence of $\left\{x_{t}\right\}$ in a Hilbert space for a subclass of nonexpansive multimaps, that is, for maps $T$ satisfying $T z=\{z\}$. Later on, Kim and Jung [13] extended López Acedo and $\mathrm{Xu}$ 's result to a Banach space having a sequentially continuous duality map. Sahu 20] discussed this problem in a uniformly convex Banach space having a uniformly Gâteaux differentiable norm. It was noted by Jung [9] that the condition that $T z=\{z\}$ should be added in the main results of Sahu [20]. Very recently, Jung [9] gave the strong convergence of $\left\{x_{t}\right\}$ defined by $x_{t} \in t T x_{t}+(1-t) u, u \in D$ for the nonexpansive nonself multimap $T$ in a uniformly convex or reflexive Banach space having a uniformly Gâteaux differentiable norm. More precisely, he obtained the following extensions of Browder's theorem.

Theorem J1 ([9], Theorem 1). Let E be uniformly convex Banach space having a uniformly Gâteaux differentiable norm, $D$ a nonempty closed convex subset of $E$, and $T: D \rightarrow K(E)$ a nonexpansive nonself multimap. Suppose that $D$ is a nonexpansive retract of $E$. Suppose that $T y=\{y\}$ for any fixed point $y$ of $T$ and that for each $u \in D$ and $t \in(0,1)$, the contraction $G_{t}$ defined by $G_{t} x=t T x+(1-t) u$ has a fixed point $x_{t} \in D$. Then $T$ has a fixed point if and only if $\left\{x_{t}\right\}$ remains bounded as $t \rightarrow 1$ and in this case, $\left\{x_{t}\right\}$ converges strongly as $t \rightarrow 1$ to a fixed point of $T$.

Theorem J2 (9, Theorem 2). Let $E$ be a reflexive Banach space having a uniformly Gâteaux differentiable norm, $D$ a nonempty closed convex subset of $E$, and $T: D \rightarrow K C(E)$ a nonexpansive nonself multimap satisfying the inwardness condition, where $K C(E)$ denotes the family of compact convex subsets of E. Suppose that every closed bounded convex subset of $D$ is compact. If $F(T) \neq \emptyset$ and Ty $=\{y\}$ for any $y \in F(T)$, then $\left\{x_{t}\right\}$, defined by $x_{t} \in t T x_{t}+(1-t) u$, converges strongly as $t \rightarrow 1$ to a fixed point of $T$.

It is still an open question whether the assumption $T y=\{y\}$ in Theorems J1 and J2 can be omitted (see, Jung [9]).

It seems that, in view of Pietramala's example [17] (see also Jung [9]), it is almost impossible to completely omit this assumption for nonexpansive multimaps. 


\section{Our concern now is the following:}

Is it possible to extend Browder's theorem to a class of multimaps under mild conditions? In other words: Can an approximating sequence be constructed which converges to a fixed point of a multimap under some mild conditions?

Let $D$ be nonempty closed convex subset of a Banach space $E$. Let $T: D \rightarrow$ $K(E)$ be a multimap and

$$
P_{T}(x)=\left\{u_{x} \in T x:\left\|x-u_{x}\right\|=d(x, T x)\right\} .
$$

Then $P_{T}: D \rightarrow K(E)$ is nonempty and compact for every $x \in D$.

Instead of

$$
G_{t} x=t T x+(1-t) u, u \in D,
$$

we consider, for $t \in(0,1)$,

$$
S_{t} x:=t P_{T}(x)+(1-t) u, u \in D .
$$

Notice that $S_{t} x \subseteq G_{t} x$ for all $x \in D$ and if $P_{T}$ is nonexpansive and $T$ is weakly inward, then $S_{t}$ is a weakly inward contraction. Now Theorem 1 of Lim [14] guarantees that $x_{t}$ is a fixed point of $S_{t}$, that is,

$$
x_{t} \in t P_{T}\left(x_{t}\right)+(1-t) u \subseteq t T\left(x_{t}\right)+(1-t) u .
$$

If $T$ is single-valued, then (1.3) is reduced to

$$
x_{t}=t T\left(x_{t}\right)+(1-t) u \text {. }
$$

It is our purpose in this paper to prove the strong convergence of $\left\{x_{t}\right\}$ defined by (1.3) (i) for a nonself multimap in a uniformly convex Banach space having a uniformly Gâteaux differentiable norm; (ii) for an inward multimap in a reflexive Banach space having a uniformly Gâteaux differentiable norm. This provides an affirmative answer to the above Question as well as gives a partial answer to Jung's Question. Our results unify, extend and complement several known results including those of Browder [3], Jung [9], Jung and Kim [10, 11], Sahu 20], Xu [27] and $\mathrm{Xu}$ and $\mathrm{Yin}$ 29].

\section{Preliminaries}

Let $S:=\{x \in E:\|x\|=1\}$ denote the unit sphere of the Banach space $E$. The space $E$ is said to have a Gâteaux differentiable norm if the limit

$$
\lim _{n \rightarrow \infty} \frac{\|x+t y\|-\|x\|}{t}
$$

exists for each $x, y \in S$, and we call $E$ smooth; $E$ is said to have a uniformly Gâteaux differentiable norm if for each $y \in S$ the limit $(*)$ is attained uniformly for $x \in S$. Furthermore, $E$ is said to be uniformly smooth if the limit $(*)$ exists uniformly for $(x, y) \in S \times S$. We denote by $J$ the normalized duality mapping from $E$ to $2^{E^{*}}$ defined by

$$
J x:=\left\{f^{*} \in E^{*}:\left\langle x, f^{*}\right\rangle=\|x\|^{2}=\left\|f^{*}\right\|^{2}\right\},
$$

where $\langle.,$.$\rangle denotes the generalized duality pairing. It is well known [4] that if E$ is smooth, then any duality mapping on $E$ is single-valued, and if $E$ has a uniformly Gâteaux differentiable norm, then the duality mapping is norm-to-weak* uniformly continuous on bounded sets.

The inward set of $D \subseteq E$ at $x$ is defined by

$$
I_{D}(x)=\{z \in E: z=x+\lambda(y-x): y \in D, \lambda \geq 0\} .
$$


It is known that if $D$ is convex, then the closure of $I_{D}(x)$ is $\overline{I_{D}(x)}=x+T_{D}(x)$ for any $x \in D$, where

$$
T_{D}(x)=\left\{y \in E: \liminf _{\lambda \rightarrow 0^{+}} \frac{d(x+\lambda y, D)}{\lambda}=0\right\} .
$$

A multimap $T: D \longrightarrow C B(E)$ is said to satisfy the (i) weak inwardness condition if $T x \subset \overline{I_{D}(x)}$ for all $x \in D$; (ii) inwardness condition if $T x \subset I_{D}(x)$ for all $x \in D$. For details, we refer to [5].

A mapping $T: D \rightarrow C B(E)$ is *-nonexpansive (8]) if for all $x, y \in D$ and $u_{x} \in T(x)$ with $d\left(x, u_{x}\right)=\inf \{d(x, z): z \in T(x)\}$, there exists $u_{y} \in T(y)$ with $d\left(y, u_{y}\right)=\inf \{d(y, w): w \in T(y)\}$ such that

$$
d\left(u_{x}, u_{y}\right) \leq d(x, y) .
$$

It is known that $*$-nonexpansiveness is different from nonexpansiveness for multimaps. There are some $*$-nonexpansive multimaps which are not nonexpansive and some nonexpansive multimaps which are not $*$-nonexpansive 28 .

Let $D \subseteq E$ be closed and convex. A mapping $Q$ of $E$ into $E$ is said to be a retraction if $Q^{2}=Q$. If a mapping $Q$ is a retraction, then $Q(z)=z$ for every $z \in$ $R(Q)$, where $R(Q)$ is the range of $Q$. A subset $K$ of $E$ is said to be a nonexpansive retract of $E$ if there exists a nonexpansive retraction of $E$ onto $K$. If $E$ is a Hilbert space, the proximity map is a sunny nonexpansive retraction from $H$ to any closed convex subset of $H$.

We shall let LIM be a Banach limit. Recall that LIM $\in\left(\ell^{\infty}\right)^{*}$ such that $\|\mathrm{LIM}\|=$ 1, $\liminf \operatorname{in}_{n \rightarrow \infty} a_{n} \leq \operatorname{LIM}_{n} a_{n} \leq \limsup _{n \rightarrow \infty} a_{n}$, and $\operatorname{LIM}_{n} a_{n}=\operatorname{LIM}_{n} a_{n+1}$ for all $\left\{a_{n}\right\}_{n} \in \ell^{\infty}$.

In what follows, we shall make use of the following lemmas.

Lemma 2.1 (see, e.g., [6, 25]). Let $E$ be a Banach space having a uniformly Gâteaux differentiable norm and $D$ a nonempty closed convex subset of $E$. Let $\left\{x_{n}\right\}$ be a bounded sequence in E, LIM be a Banach limit, and $u \in D$. Then the following are equivalent:

(i) $\operatorname{LIM}_{n}\left\|x_{n}-u\right\|^{2}=\min _{y \in D} \operatorname{LIM}_{n}\left\|x_{n}-y\right\|^{2}$;

(ii) $\operatorname{LIM}_{n}\left\langle x-u, J\left(x_{n}-u\right)\right\rangle \leq 0$ for all $y \in D$.

Lemma 2.2 (see, e.g., [18, 23]). Let $E$ be a uniformly convex Banach space, $D$ a nonempty closed convex subset of $E$, and $\left\{x_{n}\right\}$ a bounded sequence in $E$. Then the set

consists of one point.

$$
C=\left\{u \in D: \operatorname{LIM}_{n}\left\|x_{n}-u\right\|^{2}=\min _{y \in D} \operatorname{LIM}_{n}\left\|x_{n}-y\right\|^{2}\right\}
$$

Lemma 2.3 (see, e.g., 26]). Let $D$ be a nonempty compact convex subset of a Banach space $E$ and $T: D \rightarrow K C(E)$ a nonexpansive multimap satisfying the boundary condition:

$$
T x \cap \overline{I_{D}(x)} \neq \emptyset, \quad x \in D .
$$

Then $T$ has a fixed point.

\section{MAin Results}

Theorem 3.1. Let $E$ be a uniformly convex Banach space having a uniformly Gâteaux differentiable norm, $D$ a nonempty closed convex subset of $E$, and $T$ : $D \rightarrow K(E)$ a nonself multimap such that $P_{T}$ is nonexpansive. Suppose that $D$ is a 
nonexpansive retract of $E$ and that for each $u \in D$ and $t \in(0,1)$, the contraction $S_{t}$ defined by $S_{t} x=t P_{T} x+(1-t) u$ has a fixed point $x_{t} \in D$. Then $T$ has a fixed point if and only if $\left\{x_{t}\right\}$ remains bounded as $t \rightarrow 1$; in this case, $\left\{x_{t}\right\}$ converges strongly as $t \rightarrow 1$ to a fixed point of $T$.

Proof. Given any $x_{t} \in D$, we can find some $y_{t} \in P_{T}\left(x_{t}\right)$ such that

$$
x_{t}=t y_{t}+(1-t) u \text {. }
$$

Notice that $P_{T}(y)=\{y\}$ whenever $y$ is a fixed point of $T$. Let $p \in F(T)$. Then $p \in P_{T}(p)$, and thus we obtain for all $t \in(0,1)$ that

$$
\begin{aligned}
\left\|y_{t}-p\right\| & =d\left(y_{t}, P_{T}(p)\right) \\
& \leq H\left(P_{T}\left(x_{t}\right), P_{T}(p)\right) \\
& \leq\left\|x_{t}-p\right\| .
\end{aligned}
$$

We thus have

$$
\begin{aligned}
\left\|x_{t}-p\right\| & =\left\|t y_{t}+(1-t) u-p\right\| \\
& \leq t\left\|y_{t}-p\right\|+(1-t)\|u-p\| \\
& \leq t\left\|x_{t}-p\right\|+(1-t)\|u-p\|
\end{aligned}
$$

and so

$$
\left\|x_{t}-p\right\| \leq\|u-p\|
$$

for $t \in(0,1)$. Hence $\left\{x_{t}\right\}$ is uniformly bounded.

Suppose that $\left\{x_{t}\right\}$ remains bounded as $t \rightarrow 1$. Now we show that $F(T) \neq \emptyset$ and $x_{t}$ converges to a fixed point of $T$ as $t \rightarrow 1$. Let $t_{n} \rightarrow 1$ and set $x_{n}:=x_{t_{n}}$. Define the mapping $\phi: E \longrightarrow \mathbb{R}$ by

$$
\phi(x):=\operatorname{LIM}_{n}\left\|x_{n}-x\right\|^{2} .
$$

Since $E$ is reflexive, $\phi(x) \rightarrow \infty$ as $\|x\| \rightarrow \infty, \phi$ is continuous and convex, it follows that $\phi$ attains its infimum over $D$ at $p$ (say) ([1, p. 79) and so the set $C:=\left\{y \in D: \phi(y)=\inf _{x \in D} \phi(x)\right\} \neq \emptyset$. C is also a closed, bounded and convex subset of $D$. Let $Q$ be a nonexpansive retraction of $E$ onto $D$. Then

$$
\begin{aligned}
\phi(y) & =\operatorname{LIM}_{n}\left\|x_{n}-y\right\|^{2} \\
& \geq \operatorname{LIM}_{n}\left\|Q x_{n}-Q y\right\|^{2} \\
& =\operatorname{LIM}_{n}\left\|x_{n}-Q y\right\|^{2} \\
& \geq \operatorname{LIM}_{n}\left\|x_{n}-p\right\|^{2}=\phi(p)
\end{aligned}
$$

for any $y \in E$. This implies that $p$ is the global minimum point over all of $E$. Furthermore, $p$ is unique by Lemma 2.2. Notice that $x_{n}=t_{n} y_{n}+\left(1-t_{n}\right) u$ for some $y_{n} \in P_{T}\left(x_{n}\right)$ and $\left\|x_{n}-y_{n}\right\| \rightarrow 0$. We can find $w_{n} \in P_{T}(p), n \geq 1$, by compactness of $P_{T}(p)$ such that

$$
\begin{aligned}
\left\|y_{n}-w_{n}\right\| & =d\left(y_{n}, P_{T}(p)\right) \\
& \leq H\left(P_{T}\left(x_{n}\right), P_{T}(p)\right) \\
& \leq\left\|x_{n}-p\right\| .
\end{aligned}
$$

The sequence $\left\{w_{n}\right\}$ has a convergent subsequence, which we denote again by $\left\{w_{n}\right\}$, with $w_{n} \rightarrow w \in P_{T}(p)$. So

$$
\begin{aligned}
\operatorname{LIM}_{n}\left\|x_{n}-w\right\|^{2} & \leq \operatorname{LIM}_{n}\left\|y_{n}-w_{n}\right\|^{2} \\
& \leq \operatorname{LIM}_{n}\left\|x_{n}-p\right\|^{2} .
\end{aligned}
$$


But $p$ is the unique global minimum. Therefore $p=w \in P_{T}(p) \subseteq T p$ and hence $F(T) \neq \emptyset$. We have also that $P_{T}(p)=\{p\} \subseteq C$. Moreover, we have from (3.1) that

$$
\begin{aligned}
\left\langle x_{n}-y_{n}, j\left(x_{n}-p\right)\right\rangle & =\left\langle x_{n}-p+p-y_{n}, j\left(x_{n}-p\right)\right\rangle \\
& \geq\left\|x_{n}-p\right\|^{2}-\left\|y_{n}-p\right\|\left\|x_{n}-p\right\| \geq 0
\end{aligned}
$$

and so

$$
0 \leq\left\langle x_{n}-y_{n}, j\left(x_{n}-p\right)\right\rangle=\left(1-t_{n}\right)\left\langle u-y_{n}, j\left(x_{n}-p\right)\right\rangle .
$$

This together with $\left\|x_{n}-y_{n}\right\| \rightarrow 0$ imply that

$$
\operatorname{LIM}_{n}\left\langle x_{n}-u, j\left(x_{n}-p\right)\right\rangle \leq 0 .
$$

Also, by Lemma 2.1, we have

$$
\operatorname{LIM}_{n}\left\langle x-p, j\left(x_{n}-p\right)\right\rangle \leq 0
$$

for all $x \in D$. In particular,

$$
\operatorname{LIM}_{n}\left\langle u-p, j\left(x_{n}-p\right)\right\rangle \leq 0 .
$$

Thus using (3.2) and (3.3) we find that $\operatorname{LIM}_{n}\left\|x_{n}-p\right\|=0$. Therefore, there exists a subsequence $\left\{x_{n_{k}}\right\}$ of $\left\{x_{n}\right\}$ such that $x_{n_{k}} \rightarrow p$ as $k \rightarrow \infty$. Assume that there is another subsequence $\left\{x_{n_{l}}\right\}$ of $\left\{x_{n}\right\}$ such that $x_{n_{l}} \rightarrow q$ as $l \rightarrow \infty$. Since $d\left(x_{n_{l}}, P_{T}\left(x_{n_{l}}\right)\right) \leq\left(1-t_{n_{l}}\right)\left\|u-y_{n_{l}}\right\| \rightarrow 0$ as $l \rightarrow \infty$, it follows that $d\left(q, P_{T}(q)\right)=0$ and so $q \in P_{T}(q) \subseteq T(q)$. Notice that $P_{T}(q)=\{q\}$. With $x_{n_{k}} \rightarrow p$, it follows from (3.2) that

$$
\langle p-u, j(p-q)\rangle \leq 0 .
$$

Also, with $x_{n_{l}} \rightarrow q$, we have that

$$
\langle q-u, j(q-p)\rangle \leq 0 .
$$

Inequalities (3.4) and (3.5) yield that

$$
\|p-q\|^{2} \leq 0
$$

which implies that $p=q$. Thus $x_{n} \rightarrow p$ as $n \rightarrow \infty$. This completes the proof.

It is known that a nonempty closed convex subset of a Hilbert space is a nonexpansive retract with the proximity map as a nonexpansive retraction. Thus, we have the following corollary.

Corollary 3.2. Let $E$ be a Hilbert space, $D$ a nonempty closed convex subset of $E$, and $T: D \rightarrow K(E)$ a nonself multimap such that $P_{T}$ is nonexpansive. Suppose that for each $u \in D$ and $t \in(0,1)$, the contraction $S_{t}$ defined by $S_{t} x=t P_{T} x+(1-t) u$ has a fixed point $x_{t} \in D$. Then $T$ has a fixed point if and only if $\left\{x_{t}\right\}$ remains bounded as $t \rightarrow 1$ and in this case $\left\{x_{t}\right\}$ converges strongly as $t \rightarrow 1$ to a fixed point of $T$.

By definition of the Hausdorff metric, we obtain that if $T$ is $*$-nonexpansive, then $P_{T}$ is nonexpansive. Hence, we have the following corollary.

Corollary 3.3. Let $E$ be a uniformly convex Banach space having a uniformly Gâteaux differentiable norm, $D$ a nonempty closed convex subset of $E$, and $T$ : $D \rightarrow K(E)$ a nonself $*$-nonexpansive multimap. Suppose that $D$ is a nonexpansive retract of $E$ and that for each $u \in D$ and $t \in(0,1)$, the contraction $S_{t}$ defined by $S_{t} x=t P_{T} x+(1-t) u$ has a fixed point $x_{t} \in D$. Then $T$ has a fixed point if and 
only if $\left\{x_{t}\right\}$ remains bounded as $t \rightarrow 1$ and in this case, $\left\{x_{t}\right\}$ converges strongly as $t \rightarrow 1$ to a fixed point of $T$.

It is well known that every nonempty closed convex subset $D$ of a uniformly convex Banach space $E$ is Chebyshev, i.e., for every $x \in E$ there is a unique element $u \in D$ such that $\|x-u\|=\inf \{|| x-v \|: v \in D\}$. Thus, we have the following corollary.

Corollary 3.4. Let $E$ be a uniformly convex Banach space having a uniformly Gâteaux differentiable norm, $D$ a nonempty closed convex subset of $E$, and $T$ : $D \rightarrow K C(E)$ a nonself multimap such that $P_{T}$ is nonexpansive. Suppose that $D$ is a nonexpansive retract of $E$ and that for each $u \in D$ and $t \in(0,1)$, the contraction $S_{t}$ defined by $S_{t} x=t P_{T} x+(1-t) u$ has a fixed point $x_{t} \in D$. Then $T$ has a fixed point if and only if $\left\{x_{t}\right\}$ remains bounded as $t \rightarrow 1$ and in this case, $\left\{x_{t}\right\}$ converges strongly as $t \rightarrow 1$ to a fixed point of $T$.

Proof. In this case, $T x$ is Chebyshev for each $x \in D$ and so $P_{T}$ is a selector of $T$ and $P_{T}$ is a single-valued nonexpansive map. The result now follows from Theorem 3.1 .

Corollary 3.5. Let $E$ be a uniformly convex Banach space having a uniformly Gâteaux differentiable norm, $D$ a nonempty closed convex subset of $E$, and $T$ : $D \rightarrow K C(E)$ a nonself $*$-nonexpansive multimap. Suppose that $D$ is a nonexpansive retract of $E$ and that for each $u \in D$ and $t \in(0,1)$, the contraction $S_{t}$ defined by $S_{t} x=t P_{T} x+(1-t) u$ has a fixed point $x_{t} \in D$. Then $T$ has a fixed point if and only if $\left\{x_{t}\right\}$ remains bounded as $t \rightarrow 1$ and in this case, $\left\{x_{t}\right\}$ converges strongly as $t \rightarrow 1$ to a fixed point of $T$.

We know that if $D$ is a nonempty closed bounded convex subset of a uniformly convex Banach space $E$ and $T: D \rightarrow K(E)$ is a nonexpansive mapping satisfying the weak inwardness condition, then $T$ has a fixed point (see [26]). As a consequence, under this condition, the contraction $S_{t}$ defined by $S_{t} x=t P_{T} x+(1-t) u$ has a fixed point $x_{t} \in D$. Thus, we have the following results.

Corollary 3.6. Let $E$ be a uniformly convex Banach space having a uniformly Gâteaux differentiable norm, $D$ a nonempty closed bounded convex subset of $E$, and $T: D \rightarrow K(E)$ a nonself multimap satisfying the weak inwardness condition such that $P_{T}$ is nonexpansive. Suppose that $D$ is a nonexpansive retract of $E$. Then $\left\{x_{t}\right\}$ defined by (1.3) converges strongly as $t \rightarrow 1$ to a fixed point of $T$.

Corollary 3.7. Let $E$ be a Hilbert space, $D$ a nonempty closed bounded convex subset of $E$, and $T: D \rightarrow K C(E)$ a nonself multimap satisfying the weak inwardness condition such that $P_{T}$ is nonexpansive. Then $\left\{x_{t}\right\}$ defined by (1.3) converges strongly as $t \rightarrow 1$ to a fixed point of $T$.

Remark 3.8. (1) We now give an example of a multimap $T$ with $P_{T}$ nonexpansive. Let $D=[0, \infty)$ and $T$ be defined by $T x=[x, 2 x]$ for $x \in D([28)$. Then $P_{T}(x)=\{x\}$ for $x \in D$. Observe that $T$ is $*$-nonexpansive but not nonexpansive (see [28]).

(2) Theorem 3.1 extends Theorem 1 and Corollary 1 of Jung and Kim [10], Corollary 2 of Jung and Kim [11] and Theorem 1 and Corollary 2 of Xu and Yin [29] to multimaps. It also improves and complements Theorem J1, Theorem 4.1 of Kim and Jung 13 and Theorem 1 of Sahu [20].

(3) Our results apply to $L^{p}$ spaces and $l^{p}$ spaces for $1<p<\infty$. 
Theorem 3.9. Let $E$ be a reflexive Banach space having a uniformly Gâteaux differentiable norm, $D$ a nonempty closed convex subset of $E$, and $T: D \rightarrow K C(E)$ a nonself multimap satisfying the inwardness condition such that $P_{T}$ is nonexpansive. Suppose that every closed bounded convex subset of $D$ is compact and $P_{T}$ has a fixed point. Then $\left\{x_{t}\right\}$ defined by (1.3) converges strongly as $t \rightarrow 1$ to a fixed point of $T$.

Proof. Let $p \in P_{T}(p)$. Then, as in the proof of Theorem 3.1, $\left\{x_{t}\right\}$ remains bounded as $t \rightarrow 1$. Now let $t_{n} \rightarrow 1$ and set $x_{n}:=x_{t_{n}}$. Define the mapping $\phi: E \longrightarrow \mathbb{R}$ by

$$
\phi(x):=\operatorname{LIM}_{n}\left\|x_{n}-x\right\|^{2}, \forall x \in D
$$

and set

$$
C:=\left\{y \in D: \phi(y)=\inf _{x \in D} \phi(x)\right\} \neq \emptyset .
$$

$C$ is also a closed, bounded and convex subset of $D$. By assumption $C$ is compact. Clearly, $P_{T}$ satisfies the inwardness condition. Essentially the same arguments as in Theorem 2 of Jung [9] yield that

$$
P_{T}(x) \cap I_{C}(x) \neq \emptyset, \quad x \in C .
$$

Now Lemma 2.3 guarantees that there exists $p \in C$ such that $p \in P_{T}(p) \subseteq T p$ and so $P_{T}(p)=\{p\}$. As in the proof of Theorem 3.1, $\left\{x_{t}\right\}$ converges strongly as $t \rightarrow 1$ to $p$.

Corollary 3.10. Let $E$ be a reflexive Banach space having a uniformly Gâteaux differentiable norm, $D$ a nonempty closed convex subset of $E$, and $T: D \rightarrow K C(E)$ a nonself *-nonexpansive multimap satisfying the inwardness condition. Suppose that every closed bounded convex subset of $D$ is compact and $P_{T}$ has a fixed point. Then $\left\{x_{t}\right\}$ defined by (1.3) converges strongly as $t \rightarrow 1$ to a fixed point of $T$.

Corollary 3.11. Let $E$ be a uniformly smooth Banach space, $D$ a nonempty closed convex subset of $E$, and $T: D \rightarrow K C(E)$ a nonself multimap satisfying the inwardness condition such that $P_{T}$ is nonexpansive. Suppose that every closed bounded convex subset of $D$ is compact and $P_{T}$ has a fixed point. Then $\left\{x_{t}\right\}$ defined by (1.3) converges strongly as $t \rightarrow 1$ to a fixed point of $T$.

Corollary 3.12. Let $E$ be a reflexive Banach space having a uniformly Gâteaux differentiable norm, $D$ a nonempty compact convex subset of $E$, and $T: D \rightarrow K C(E)$ a nonself multimap satisfying the inwardness condition such that $P_{T}$ is nonexpansive. Suppose that $P_{T}$ has a fixed point. Then $\left\{x_{t}\right\}$ defined by (1.3) converges strongly as $t \rightarrow 1$ to a fixed point of $T$.

Remark 3.13. (1) Theorem 3.9 extends Theorem 1 and Corollary 1 of Jung and Kim [1] and Theorem 1 of $\mathrm{Xu}$ [27] to multimaps.

(2) Recently it has been shown by Shahzad and Lone 21] that if $D$ is a nonempty closed bounded convex subset of a Banach space $E$ such that $\epsilon_{\alpha}(E)<1$ and $T: D \rightarrow K C(E)$ is a $*$-nonexpansive, 1 - $\chi$-contractive multimap satisfying the inwardness condition, then $T$ has a fixed point. So one can relax the assumption that $F(T) \neq \emptyset$ in certain situations. 


\section{REFERENCES}

[1] V. Barbu and Th. Precupanu, Convexity and Optimization in Banach Spaces, Editura Academiei R. S. R., Bucharest, 1978.

[2] V. Berinde, Iterative Approximation of Fixed Points, Editura Efemeride, Baia Mare, 2002. MR1995230 (2004i:47102)

[3] F. E. Browder, Convergence of approximants to fixed points of nonexpansive mappings in Banach spaces, Arch. Rational Mech. Anal., 24 (1967), 82-90. MR0206765 (34:6582)

[4] I. Ciorenescu, Geometry of Banach spaces, Duality Mapping and Nonlinear Problems, Kluwer Academic Publishers, 1990. MR1079061 (91m:46021)

[5] K. Deimling, Multivalued Differential Equations, Walter de Gruyter, Berlin, 1992. MR.1189795 (94b:34026)

[6] K. S. Ha and J. S. Jung, Strong convergence theorems for accretive operators in Banach spaces, J. Math. Anal. Appl., 147 (1990), 330-339. MR.1050208(91k:47132)

[7] B. Halpern, Fixed points of nonexpansive maps, Bull. Amer. Math. Soc., 73 (1967), 957-961. MR0218938 (36:2022)

[8] T. Hussain and A. Latif, Fixed points of multivalued nonexpansive maps, Math. Japonica, 33 (1988), 385-391. MR956852 (89i:47107)

[9] J. S. Jung, Strong convergence theorems for multivalued nonexpansive nonself-mappings in Banach spaces, Nonlinear Anal., 66 (2006), 2345-2354.

[10] J. S. Jung and S. S. Kim, Strong convergence theorems for nonexpansive nonself-mappings in Banach spaces, Nonlinear Anal., 33 (1998), 321-329. MR1617964 (99h:47068)

[11] J. S. Jung and T. H. Kim, Strong convergence of approximating fixed points for nonexpansive nonself-mappings in Banach spaces, Kodai Math. J., 21 (1998), 259-272. MR.1661635 (99k:47134)

[12] G. E. Kim and W. Takahashi, Strong convergence theorems for nonexpansive nonselfmappings in Banach spaces, Nihonkai Math. J., 7 (1996), 63-72. MR1389684 (98m:47088)

[13] T. H. Kim and J. S. Jung, Approximating fixed points of nonlinear mappings in Banach spaces. Proceedings of Workshop on Fixed Point Theory (Kazimierz Dolny, 1997), Ann. Univ. Mariae Curie-Sktodowska Sect. A 51 (1997), 149-165. MR.1666175(2000a:47119)

[14] T. C. Lim, A fixed point theorem for weakly inward multivalued contractions, J. Math. Anal. Appl., 247 (2000), 323-327. MR1766943 (2001d:47088)

[15] G. Lopez Acedo and H. K. Xu, Remarks on multivalued nonexpansive mappings, Soochow J. Math., 21 (1995), 107-115. MR.1319554 (96f:47108)

[16] S. Nadler, Multivalued contraction mappings, Pacific J. Math., 30 (1969), 475-488. MR0254828 (40:8035)

[17] P. Pietramala, Convergence of approximating fixed point sets for multivalued nonexpansive mappings, Comment. Math. Univ. Carolinae, 32 (1991), 697-701. MR1159816 (93f:47075)

[18] S. Reich, Nonlinear semigroups, holomorphic mappings, and integral equations, in: Proc. Symp. Pure Math., Vol. 405, Part 2, Amer. Math. Soc. Providence, RI, 1986, pp. 287-292. MR843617 (87j:47101)

[19] S. Reich, Strong convergence theorems for resolvents of accretive operators in Banach spaces, J. Math. Anal Appl., 75 (1980), 287-292. MR576291 (82a:47050)

[20] D. R. Sahu, Strong convergence theorems for nonexpansive type and non-self multi-valued mappings, Nonlinear Anal., 37 (1999), 401-407. MR1694398(2000d:47076)

[21] N. Shahzad and A. Lone, Fixed points of multimaps which are not necessarily nonexpansive, Fixed Point Theory and Appl., 2005:2 (2005), 169-176. MR2199937 (2006j:47097)

[22] S. P. Singh and B. Watson, On approximating fixed points, Proc. Symp. Pure Math., 45 (1988), 393-395. MR843624(87h:47134)

[23] W. Takahashi and D. H. Jeong, Fixed point theorem for nonexpansive semigroups on Banach spaces, Proc. Amer. Math. Soc., 122 (1994), 1175-1179. MR1223268 (95b:47087)

[24] W. Takahashi and G. E. Kim, Strong convergence of approximants to fixed points of nonexpansive nonself-mappings in Banach spaces, Nonlinear Anal., 32 (1998), 447-454. MR1610606 (99h:47063)

[25] W. Takahashi and Y. Ueda, On Reich's strong convergence theorems for resolvents of accretive operators, J. Math. Anal. Appl., 104 (1984), 546-553. MR766150 (86c:47070)

[26] H. K. Xu, Multivalued nonexpansive mappings in Banach spaces, Nonlinear Anal., 43 (2001), 693-706. MR1808203 (2002b:47122) 
[27] H. K. Xu, Approximating curves of nonexpansive nonself-mappings in Banach spaces, C. $R$. Acad. Sci. Paris, Ser. I, 325 (1997), 179-184. MR.1467068(98i:47052)

[28] H. K. Xu, On weakly nonexpansive and *-nonexpansive multivalued mappings, Math. Japonica, 36 (1991), 441-445. MR1109228 (92e:54052)

[29] H. K. Xu and X. M. Yin, Strong convergence theorems for nonexpansive non-self-mappings, Nonlinear Anal., 24 (1995), 223-228. MR1312591 (95m:47115)

Department of Mathematics, King Abdul Aziz University, P.O. Box 80203, Jeddah 21589, SAUdi Arabia

E-mail address: nshahzad@kau.edu.sa

Bahir Dar University, P.O. Box. 859, Bahir Dar, Ethiopia

E-mail address: habtuzh@yahoo.com 